\title{
A note on a bronchial tree with 23 levels of bifurcation
}

\author{
Akira Nakayama
}

Published online: 4 April 2010

(C) Springer-Verlag 2010

Our recent article "Why do we have a bronchial tree with 23 levels of bifurcation?" [1] came out in Heat and Mass Transfer in 2009, in which we proposed an analytical model for the lung architecture, namely, quasi-steady onedimensional mass transfer model for the lung. In its introduction, we referred to the paper of Prof. Reis' group, namely, "Constructal theory of flow architectures of the lungs" [2] as one of the most important papers in this particular topic, explicitly stating, "Others (i.e. Prof. Reis' group) were certainly successful to some extent in relating the number of the bifurcation levels to the best oxygen access to the alveolar sacs." We believe that Prof. Reis' group certainly deserves credit for explaining for the first time the reason why we have a bronchial tree with 23 levels of bifurcation on the basis of Bejan's Constructal law.

In our paper, we mentioned the originality of our paper, stating, "Here, we shall present a simple and yet rational model for the respiratory system, namely, a quasi-steady one-dimensional mass transfer model. The model describes the mass transfer between the airway inlet and the red blood cell interior. Using this mass transfer model, we shall show that there exists the optimal number of the bifurcation levels, namely, 23, that yields the minimum overall mass transfer resistance for the mass transport from the external air to the red blood cells."

Although the conclusions drawn in the two papers, namely, the optimal number of the bifurcation levels being

The article discussed in this note can be found at doi:10.1007/s00231-008-0434-0 (2009) 45:351-354.

A. Nakayama ( $($ )

Department of Mechanical Engineering,

Shizuoka University, 3-5-1 Johoku, Naka-ku,

Hamamatsu 432-8561, Japan

e-mail: tmanaka@ipc.shizuoka.ac.jp
23 , are the same, the difference in the approaches taken in the two papers is quite clear. We proposed a quasi-steady one-dimensional mass transfer model to explain the reason why we have a bronchial tree with 23 levels of bifurcation, whereas they elegantly resorted to Bejan's Constructal law with some assumptions such as isothermal and adiabatic flows with all species having the same chemical potential.

Naturally, the resulting equations giving the total number of bifurcations differ substantially from each other. Our equation requires the total pressure difference for breathing and the alveolar sac effective diffusivity, whereas theirs requires quantities such as the gas constant, absolute temperature, the oxygen relative concentration at the entrance of the trachea and that in the external air. Despite of the substantial difference in appearance of the two equations, both equations give the same optimal number of the bifurcation levels. This, however, is not surprising, since all rational scientific approaches should lead to the same fact in nature.

\section{References}

1. Nakayama A, Kuwahara F, Sano Y (2009) Why do we have a bronchial tree with 23 levels of bifurcation? Heat Mass Transf 45(3):351-354

2. Reis AH, Miguel AF, Aydin V (2004) Constructal theory of flow architecture of the lungs. Med Phys 31(5):1135-1140 